\title{
Stephan Preibisch
}

\author{
Staying mobile and building a Toy-Story-related way to stitch terabyte-sized images in microscopy.
}

$\mathrm{H}$ is family owns an architectural firm and he started out studying architecture, says Stephan Preibisch, a researcher at the Berlin Institute of Medical Systems Biology (BIMSB) of the Max Delbrück Center for Molecular Medicine. But he had always liked computers and had programmed for the family firm. He switched to computer science and also grew curious about DNA.

A knock on the door of a bioinformatics institute in Leipzig landed Preibisch a project on microarray normalization.

Later, as a PhD student, he launched into bioimage analysis after seeing a prototype of a Zeiss light-sheet microscope at the Max Planck Institute of Molecular Cell Biology and Genetics (MPI-CBG) in Dresden. "I'm always drawn to challenges," he says. The microscope's gigabyte-sized file output stymied people. "It's what terabytes are today", he says. "I thought "wow I can try to address this,' that's how it all started." He has also done some consulting work for Zeiss.

Now that light-sheet microscopy or expansion microscopy give labs a view of cleared organs, entire zebrafish or fly larvae, biologists can ask entirely new types of questions, he says. "Before you wouldn't ask them because you couldn't answer them." To query their data, labs stitch their stack of micrographs together. That's where BigStitcher comes in, developed by Preibisch and, among other colleagues, David Hörl and Fabio Rojas Rujak.

BigStitcher integrates new algorithms and adapts existing ones. Unlike other stitching tools, he says, it lets users zoom in and out of terabyte sized-images so "people can interact and play with the data," he says.

Stitching might just seem a matter of moving image tiles around to fit a $3 \mathrm{D}$-image but, it's not that easy, he says. Some tiles contain little information. BigStitcher calculates the most reasonable spot for them in the stitched, aligned image. The tiles have some overlap, such as membrane intersections or nuclei, which are landmarks that BigStitcher uses for fine alignment.

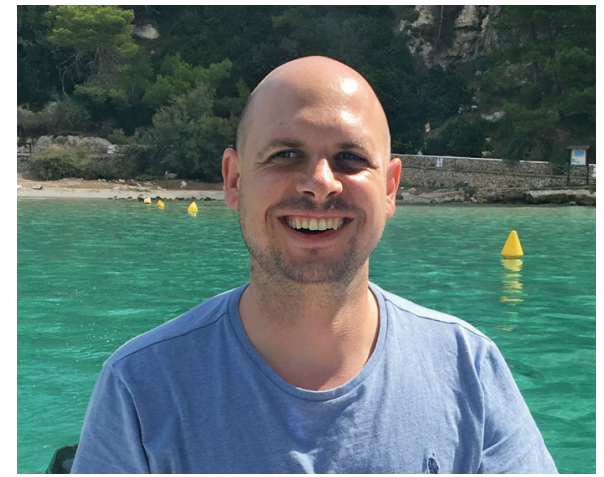

Stephan Preibisch. Credit: M. A. Costa Boleta

When stitching high-resolution images, the software has to handle chromatic and spherical aberrations. Also, a cleared sample can seem foggy in some spots, turning a fluorophore into a blurry, gray something, says Preibisch. He began wondering: "Why do we see these aberrations?" He investigated with raytracing, a technique used in animated films such as Toy Story, to follow events in a light path. "I wrote a little raytracing simulation of light propagation," he says. This helped the team algorithmically compensate for aberrations and build ways to computationally warp each pixel and stitch the tiles together more smoothly.

Preibisch completed his $\mathrm{PhD}$ research with Pavel Tomancak at MPI-CBG, then started a postdoctoral fellowship at Janelia Research Campus, in Ashburn, Virginia, with Gene Myers. A little less than a year later, Myers moved to MPI-CBG. Preibisch considered going along but, with a Human Frontier Science Program grant in hand, he decided instead to craft, as he calls it, a "delocalized post-doc" in biology and computing.

He worked with Robert Singer at Albert Einstein College of Medicine in New York, continued his project with the Myers lab and collaborated with colleagues at Janelia Research Campus. In 2015, he joined the faculty at the Max Delbrück Center. As of this October, he is returning to Janelia as a team leader.
Stephan Preibisch is a problem solver, says Tomancak about his former student, whom he calls more of a friend than a colleague. During his $\mathrm{PhD}$, and without an image-analysis background, Preibisch solved data reconstruction problems in multi-view selective plane illumination microscopy. "Stephan is incredibly efficient," says Tomancak. He balances work and life, creates "maximally usable software that helps solving biology frontier problems" and keeps tools user-friendly and applicable to tasks large and small. "I think that Stephan cannot help himself but write user-friendly software, because he is an extremely friendly and social person," says Tomancak. "We can only hope that he continues doing the 'stuff that matters' in bio-image analysis in the future."

\section{"I'm always drawn to challenges"}

In his spare time, Preibisch has enjoyed travel, tennis and jogging. As the father of a 10-month old boy, "my hobby is now our boy," he says. As the family gets ready to move, "I'm happy and sad to leave," says Preibisch about his next chapter. He's sad to leave BIMSB, but happy about his new beginning at Janelia. There will be new collaborations and older ones to continue. His friend Stephan Saalfeld, who did his $\mathrm{PhD}$ alongside Preibisch, is a Janelia researcher. Preibisch looks forward to his lab group. His advisors helped him "ask my own questions and solve them," he says. They were there when he needed help and that's the kind of PI he is now. "I really try to encourage people to find their own way."

\section{Vivien Marx}

Published online: 9 August 2019 https://doi.org/10.1038/s41592-019-0549-X

\section{References}

Hörl, D. et al. BigStitcher: reconstructing highresolution image datasets of cleared and expanded samples. Nat. Methods https://doi.org/10.1038/ s41592-019-0501-0 (2019). 